\title{
Effects of intestinal colonization by Clostridium difficile and Staphylococcus aureus on microbiota diversity in healthy individuals in China
}

Danfeng Dong ${ }^{1 \dagger}$, Qi Ni ${ }^{1 \dagger}$, Chen Wang ${ }^{1}$, Lihua Zhang ${ }^{2}$, Zhen $\mathrm{Li}^{3}$, Cen Jiang ${ }^{1}$, EnqiangMao ${ }^{4^{*}}$ and Yibing Peng ${ }^{1 *}$

\begin{abstract}
Background: Intestinal colonization by pathogenic bacteria is a risk factor for infection, and contributes to environmental contamination and disease dissemination. Alteration of gut microbiota also plays a pivotal role in the development of disease. Although Clostridium difficile and Staphylococcus aureus are well-recognized pathogens causing nosocomial and community infections, the intestinal colonization was not fully investigated. Herein, we explored their overall carriage rates in healthy adults from the community, and characterized the gut microbiomes of $C$. difficile and S. aureus carriers.
\end{abstract}

Methods: Fecal samples were collected from 1709 healthy volunteers from communities in Shanghai, China, and tested for the presence of C. difficile, methicillin-sensitive S. aureus (MSSA), and methicillin-resistant S. aureus (MRSA) using culture-based techniques. To explore differences in the gut microbiome, $16 \mathrm{~S}$ rRNA gene sequencing was conducted using samples from non-carriers (CH), C. difficile carriers (CCD), MRSA carriers (CM), and MSSA carriers (CS).

Results: Overall, we detected 12 C. difficile and 60 S. aureus isolates, accounting for $0.70 \%$ and $3.51 \%$ of total isolates, respectively. Eight isolates were determined to be MRSA, accounting for $13.3 \%$ of the $S$. aureus population. Sequencing data revealed that the microbial diversity and richness were similar among the four groups. However, at the phylum level, carriage of C. difficile or MRSA was associated with a paucity of Bacteroidetes and an overabundance of Proteobacteria compared with non-carriers. At the genus level, the prevalence of the genera Bacteroides, Prevotella, Faecalibacterium, and Roseburia was decreased in C. difficile-positive samples compared with the controls, while the proportion of Clostridium cluster XIVa species was increased. MRSA carriers exhibited a higher proportion of the genera Parasutterella and Klebsiella, but a decreased prevalence of Bacteroides. Compared with MSSA carriers, Klebsiella was the only genus found to be significantly enriched in MRSA carriers.

Conclusions: In healthy adults, colonization by C. difficile or S. aureus did not significantly affect gut microbiota diversity. However, the alteration of the gut microbiota composition in C. difficile carriers could indicate a predisposition to further infection. Our study provides essential data on the prevalence and effects of $C$. difficile and $S$. aureus colonization on gut microbiota composition in healthy adults.

Keywords: Clostridium difficile, Staphylococcus aureus, Intestinal colonization, Gut microbiota

\footnotetext{
* Correspondence: maoeq@yeah.net; pyb9861@sina.com

${ }^{\dagger}$ Equal contributors

${ }^{4}$ Department of Emergency Intensive Care Unit, Ruijin Hospital, Shanghai Jiaotong

University School of Medicine, No.197 Ruijin ER Road, Shanghai 200025, China

'Department of Laboratory Medicine, Ruijin Hospital, Shanghai Jiaotong

University School of Medicine, No.197 Ruijin ER Road, Shanghai 200025, China

Full list of author information is available at the end of the article
}

(c) The Author(s). 2018 Open Access This article is distributed under the terms of the Creative Commons Attribution 4.0 International License (http://creativecommons.org/licenses/by/4.0/), which permits unrestricted use, distribution, and reproduction in any medium, provided you give appropriate credit to the original author(s) and the source, provide a link to the Creative Commons license, and indicate if changes were made. The Creative Commons Public Domain Dedication waiver (http://creativecommons.org/publicdomain/zero/1.0/) applies to the data made available in this article, unless otherwise stated. 


\section{Background}

Clostridium difficile and Staphylococcus aureus are well known pathogens, causing both nosocomial and community-acquired infections. Although the clinical manifestations of these pathogens are diverse, their colonization and subsequent dissemination from the intestinal tract show a commonality $[1,2]$. C. difficile is widely accepted as the most common cause of antibiotic-associated diarrhea, and the incidence and severity of $C$. difficile infection has dramatically increased over past decades [3]. Unlike C. difficile, S. aureus gut colonization, which is a known risk factor for gastrointestinal infection [4], has not yet been well studied. During the past decade, with the pandemic rise in the incidence of methicillin-resistant $S$. aureus (MRSA) and methicillin-sensitive $S$. aureus (MSSA) infection in the community and in healthcare facilities, fecal carriage of $S$. aureus has attracted attention. For example, studies have shown that patients with intestinal colonization of MSSA and/or MRSA have a higher frequency of diarrhea and environmental contamination, as well as increased skin colonization [4, 5]. While many studies have investigated the current status of $C$. difficile, MSSA, and MRSA colonization of high-risk populations and healthcare facilities, there is limited data regarding the prevalence and colonization of these pathogens in healthy individuals in the community in China.

The diverse and abundant microbiota of the human intestine plays a crucial role in protection against pathogen colonization, as well as in overall human health [6]. However, intestinal infection or colonization by pathogens such as $S$. aureus and C. difficile is associated with disruption of the gut microbiota [7]. Numerous studies have shown that the biodiversity and community structure of the gut microbiota is altered in patients infected with $C$. difficile or $S$. aureus, and these alterations may directly lead to diseases $[6,8]$. In addition, gut dysbiosis caused by MRSA colonization is more severe than that caused by MSSA, with reduced microbial diversity and a decreased prevalence of beneficial bacterial [9]. Fecal microbiota transplantation has been successfully used to treat $C$. difficile infection and MRSA-associated enterocolitis [10], indicating that modulation of the gut microbiota may be a potentially useful approach to treat refractory infections. However, whether the intestinal bacterial communities of asymptomatic carriers are notably changed in individuals after joining a community has not yet been studied and needs to be clarified.

The main focus of this study was to estimate the prevalence of $C$. difficile and $S$. aureus in the gut of healthy adults in the communities, and to investigate whether the carriage of these pathogens was related to alterations in the gut microbiota composition.

\section{Methods}

\section{Study design and specimen collection}

The present study was conducted in four communities in Shanghai, China, between May and August 2014. Healthy individuals aged over 18 years without any acute or chronic gastrointestinal diseases were enrolled. Those who were exposed to any hospital environment or antibiotics in the previous 30 days before collection were excluded. Demographic data concerning the age and gender of the participants was collected. A total of 1709 participants were enrolled in the present study. Among them, 748 were female and 961 were male. The average age was $63(+/-16)$ years old. To better document age-related kinetics, we categorized the participants into three groups based on their ages: youth (1840 years), middle-aged ( $41-65$ years), and elderly ( $>65$ years). Fresh fecal samples were collected into sterile containers and submitted for immediate culture. All samples were stored at $-80{ }^{\circ} \mathrm{C}$ for subsequent DNA extraction.

To further characterize the intestinal microbiota of $C$. difficile- and $S$. aureus-positive individuals, we divided participants into four groups: 1) healthy individuals without any $C$. difficile or S. aureus colonization (group $\mathrm{CH}$ ), 2) individuals positive for $C$. difficile (group CCD), 3) individuals positive for MRSA (group CM), and 4) those positive for MSSA (group CS). Due to the results that there were fewer individuals positive for C.difficile and MRSA, all individuals meeting the criteria for the $C C D$ and $C M$ groups were included in the study, with participants in the $\mathrm{CH}$ and $\mathrm{CS}$ groups randomly chosen and then age and gender-matched with participants in the first two groups. Detailed information regarding the selected participants is listed in Additional file 1: Table S1.

\section{Detection of bacterial pathogens}

To improve the sensitivity of $C$. difficile detection, we aliquoted each stool sample into two parts. One aliquot was pretreated with ethanol and then directly plated onto cycloserine cefoxitin fructose agar (Oxoid Ltd., Basingstoke, UK). The other was pre-cultured in cycloserine cefoxitin fructose medium supplemented with taurocholic acid and lysozyme for $48 \mathrm{~h}$ to enrich the vegetative cells prior to plating on cycloserine cefoxitin fructose agar. Culturing was performed at $35{ }^{\circ} \mathrm{C}$ for $48 \mathrm{~h}$ in anaerobic condition. Suspected C. difficile colonies were identified based on their odor and appearance, and confirmed using a latex agglutination test (C. difficile Agglutination Test Kit; Oxoid), gluD gene detection, and a toxin A \& B test using a VIDAS Immunoanalyzer (Biomerieux, Marcy-l'Etoile, France).

To screen S. aureus isolates from stool samples, samples were plated on mannitol salt agar (Becton Dickinson) and cultured for $48 \mathrm{~h}$ at $35^{\circ} \mathrm{C}$ in aerobic condition. Suspected $S$. aureus colonies were identified based on microbiological examination. MRSA was further confirmed by Kirby-Bauer disk diffusion assay (30 mg/liter cefoxitin) 
[11] and mecA gene detection using S. aureus ATCC25923 as a negative control.

\section{DNA extraction}

Approximately $200 \mathrm{mg}$ of each fecal sample was used for genomic DNA extraction using a TIANamp Stool DNA Kit (Tiangen Biotech, Beijing, China) as per the manufacturer's instructions. A NanoDrop spectrophotometer (Thermo Fisher Scientific, Waltham, MA, USA) was used to measure the DNA concentration and A260/ A280 ratio. A PCR assay targeting the V3-V4 regions of the 16S rRNA gene was then conducted using universal primers (F: 5'-GAGAGTTTGATCCTGGCTCAG-3'; R: 5'-AAGGAGGTGATCCAGCCGCA-3') to further confirm the quality and quantity of extracted DNA.

\section{S rRNA gene sequencing and data processing}

$16 \mathrm{~S}$ rRNA gene amplification and sequencing was conducted in the Chinese National Human Genome Center (Shanghai, China). Briefly, hyper variable V3-V4 regions of the $16 \mathrm{~S}$ rRNA genes were amplified using primers V3-343F (5'-TACGGRAGGCAGCAG-3') and V4-798R (5'-AGGGTATCTAATCCT-3') with adaptor sequences (LinkerF: 5'TCGTCGGCAGCGTCAGATGTGTATAAGAGACAG-3'; LinkerR: 5'-GTCTCGTGGGCTCGGAGATGTGTATAAGAGACAG-3'), followed by sequencing using the Illumina Miseq system via standard procedures. Sequencing reads were screened to remove low quality reads, unmatched barcode sequences, and short read lengths.

Quality-trimmed sequences were clustered into one operational taxonomic unit (OTU) at a $97 \%$ similarity cutoff. Taxonomic assignment was carried out using the Ribosomal Database Project Naïve Bayesian Classifier with a cutoff value of 0.03 . The rarefaction curves were measured at an OTU level of 0.03 to evaluate the abundance between samples. Alpha diversity assessment, including community richness (abundance-based coverage estimator (ACE) richness index and Chao richness index analyses) and community diversity (Shannon diversity index and Simpson diversity index analyses), was carried out using mothur software (available from https://www.mothur.org/).

\section{Statistical analyses}

Differences in biodiversity index between four groups were compared using a one-way analysis of variance test. To analyze the relative intestinal composition at the phylum and genus level between groups, a MannWhitney test was conducted in GraphPad Prism 5. All differences were considered significant at $P<0.05$.

\section{Results}

\section{Study population}

As shown in Tables 1, 12 C. difficile and $60 \mathrm{~S}$. aureus isolates were identified, accounting for $0.70 \%$ and $3.51 \%$
Table 1 Prevalence of Clostridium difficile and Staphylococcus aureus in the intestines of healthy adults in the community in Shanghai, China, 2014

\begin{tabular}{|c|c|c|c|c|c|}
\hline \multirow{2}{*}{$\begin{array}{l}\text { Age } \\
\text { (years) }\end{array}$} & \multirow[t]{2}{*}{ No. } & \multicolumn{2}{|c|}{ C. difficile } & \multicolumn{2}{|c|}{ S. aureus } \\
\hline & & No. & $\%$ & No. & $\%$ \\
\hline $18-40$ & 130 & 0 & $0.00 \%$ & 8 & $6.15 \%$ \\
\hline $41-65$ & 837 & 3 & $0.36 \%$ & 32 & $3.82 \%$ \\
\hline$>65$ & 742 & 9 & $1.21 \%$ & 20 & $2.70 \%$ \\
\hline Overall & 1709 & 12 & $0.70 \%$ & 60 & $3.51 \%$ \\
\hline
\end{tabular}

of the total number of isolates, respectively. Of the $S$. aureus isolates, eight (13.1\%) were confirmed to be MRSA. None of the participants were found to be colonized with both $C$. difficile and $S$. aureus. Compared with the two younger groups, the elderly participants were more frequently positive for $C$. difficile. However, the prevalence of $S$. aureus decreased with age, with the highest prevalence $(6.15 \%)$ observed in youth, and the lowest $(2.7 \%)$ prevalence in the elderly (Table 1$)$.

\section{Sequencing reads and biodiversity}

We obtained a total of 1,404,279 valid sequences from the 43 selected participants after barcode trimming and primer sequencing, with an average of 32,657 sequences per sample. Subsequent analysis was performed after normalizing the sequence depth of all samples to that of the lowest sample size $(12,394)$. All samples had good index coverage (>95\%), with OTU-level rarefaction curves almost reaching a plateau, indicating sufficient sequencing depth (Additional file 2: Figure S1a). The number of OTUs for each sample ranged from 369 to 900 , with similar median numbers of OTUs across the four groups (Additional file 2: Figure S1b). Compared with the healthy controls, the $C$. difficile-positive samples had a lower average Shannon diversity index value and a higher average Simpson diversity index value; however, these differences were not significant, even when community richness was assessed using Chao and ACE index analyses (Fig. 1). The median community richness and diversity values for the MSSA- and MRSA-positive samples were similar to those of the healthy controls (Fig. 1).

\section{Microbial community structure}

The distribution of the most dominant bacterial phyla in each of the groups is listed in Fig. 2a. Although there was variation among all samples, Firmicutes and Bacteroidetes were always the predominant bacterial phyla. When analyzing the pooled samples in each group, we clearly observed that compared with the healthy controls, the CCD and CM groups had a noticeably lower proportion of Bacteroidetes (28.98\% and $26.47 \%$ respectively versus $44.20 \%$ of the controls) but a relatively higher proportion of Proteobacteria (21.36\% and 19.51\% 

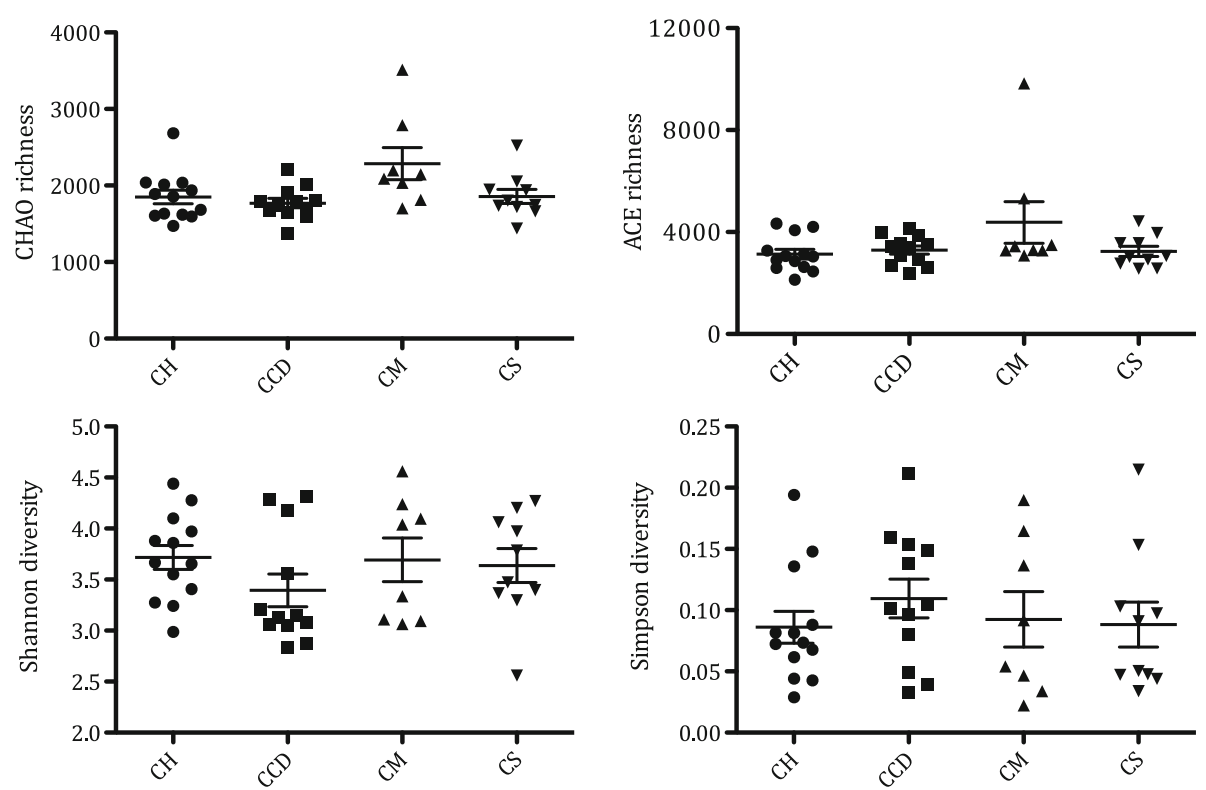

Fig. 1 Indices of microbial richness and diversity for all samples (shown in group). Data represent the mean value and standard error of each group. CH: non-carriers; CCD: Clostridium difficile carriers; CM: methicillin-resistant Staphylococcus aureus carriers; CS: methicillin-sensitive Staphylococcus aureus carriers

respectively versus $11.80 \%$ of the controls) (Fig. 2b). The distribution of bacterial phyla in the CS group was similar to that of the healthy controls, except for a decrease in the prevalence of Proteobacteria (5.61\%) (Fig. 2b).

At the genus level, all genera present at a relative abundance of $>1 \%$ are shown in Fig. 3a. In individuals positive for $C$. difficile, we observed significant decreases in the prevalence of Bacteroides and Prevotella species within the phylum Bacteroidetes. The abundance of Prevotella in the CCD group sharply declined from $5.33 \%$ to $0.03 \%$. Firmicutes, Faecalibacterium, and Roseburia sequences were found to decrease in prevalence in the CCD group, while there was a significant increase in the prevalence of Clostridium cluster XIVa. Although there were mean differences in the proportions of Escherichia/Shigella, Klebsiella, Lachnospiracea incerae sedis, Alistipes, and Fusobacterium in the gut microbiota of the CCD group, no statistical significance was found (Fig. 3b).

Surprisingly, the composition of the gut microbiota of MRSA-positive samples differed markedly from that of the MSSA-positive samples (Fig. 3c). MSSA-positive samples had a relative higher proportion of Faecalibacterium, Parabacteroides, and Haemophilus, but a lower percentage of Escherichia/Shigella. In comparison, the MRSApositive samples had a significantly higher prevalence of Klebsiella and Parabacteroides $(P<0.05)$, but a lower proportion of Bacteroides. It is also noteworthy that the relative abundance of Parasutterella species increased progressively from the $\mathrm{CH}$ to the CS samples and then to the CM samples. Klebsiella was the only genus that was significantly enriched in the MRSA-positive samples compared with the MSSA-positive samples.

Principal coordinate analysis of unweighted UniFrac distances was used to identify the potentially divergent clusters among different groups based on the microbiota structure. However, the plot showed a moderate to high degree of overlap, and failed to discriminate any differences between groups (Additional file 2: Figure S2).

\section{Discussion}

According to reports from China, the asymptomatic colonization with C.difficile was estimated to $10-20 \%$ in hepatic cirrhosis or preoperative colorectal cancer patients $[12,13]$. However, to our best knowledge, the colonization pattern of C.difficile in healthy individuals has not been well determined domestically. In the present study, we found that the gut colonization rate of C. difficile among healthy adults in the community was $0.7 \%$. This was in agreement with a report from Hesse, Germany [14], which identified a C. difficile colonization rate of $0.8 \%$ in healthy adults living outside long-term care facilities. However, the age structure of the two studies differed, with an average age of participants in the current study of 63 years, while that of the previous study was 48 years. As both studies showed that the acquisition of $C$. difficile increased with age, the overall colonization rate in Shanghai, China might be relatively lower than that in Hesse, Germany. Among the elderly, our observed colonization rate of $1.35 \%$ was similar to that reported by Rea et al. in Ireland [15], but lower than 


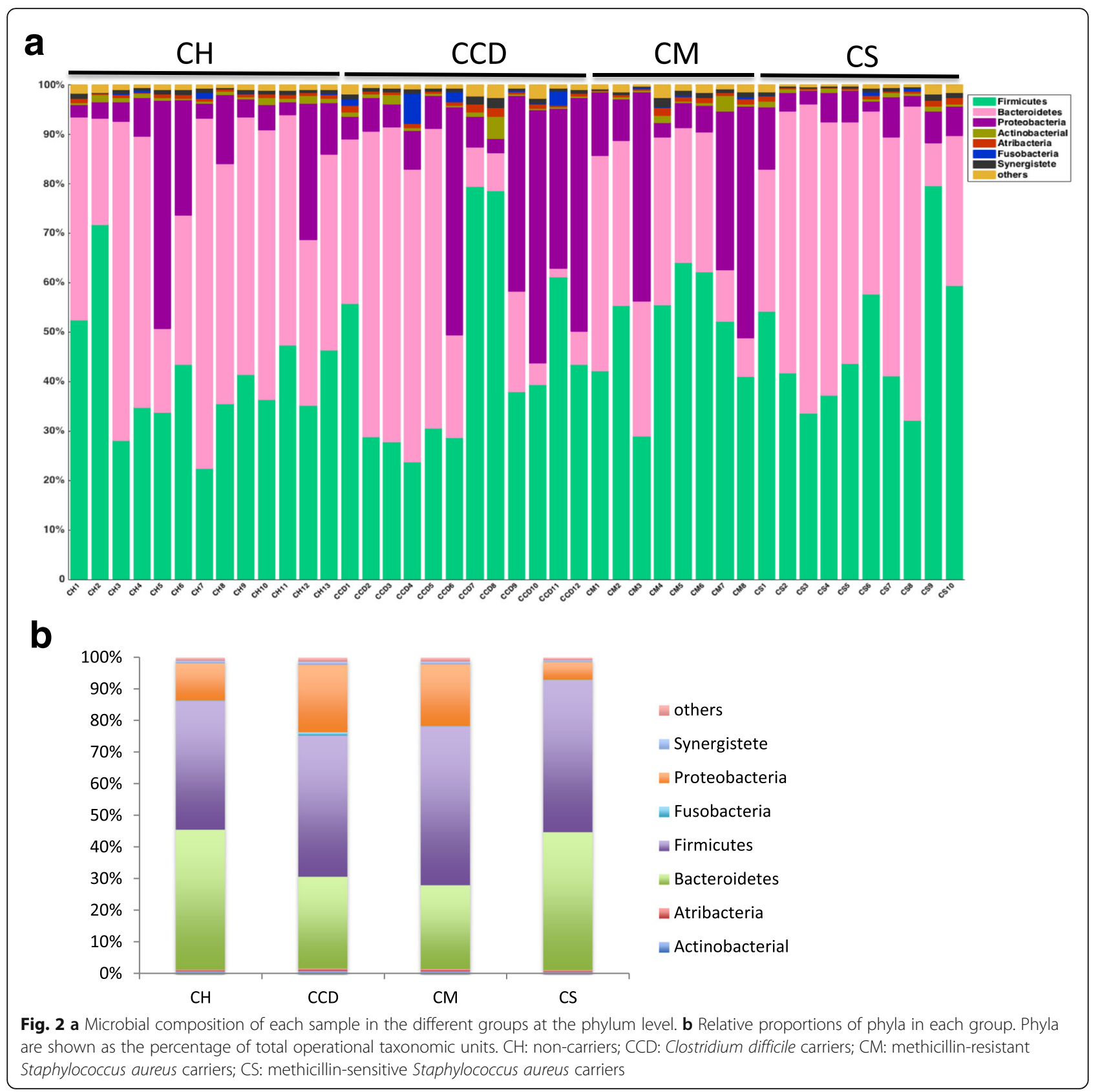

a previous report of $4 \%$ in community-dwelling elderly people in the United Kingdom [16]. As no outbreaks of C. difficile infection have yet occurred in Shanghai, it is reasonable to conclude that the colonization rate is not as high as that in European countries. However, because of the population pyramid structure and the ageing population in China, it will be important to monitor $C$. difficile colonization and infection rates, especially in community settings.

Numerous studies have shown that nasal carriage of $S$. aureus, the most common site of colonization, is a risk factor for infection [17]. However, since the epidemic- scale emergence of community-acquired MSSA and MRSA, several extra-nasal carriage sites have aroused attention. Intestinal colonization is a vital concomitant reservoir for $S$. aureus, but is poorly understood. In the present study, the overall prevalence of $S$. aureus is the gut was $3.51 \%$, with MSSA and MRSA accounting for 3 . $04 \%$ and $0.47 \%$ of all isolates, respectively. According to the meta-analysis conducted by Gagnaire et al. involving 712 studies [18], the intestinal carriage rate in healthy adults was estimated to be approximately $13.8 \%$ for $S$. aureus and $1.4 \%$ for MRSA, much higher than those detected in the current study. The studies included in the 


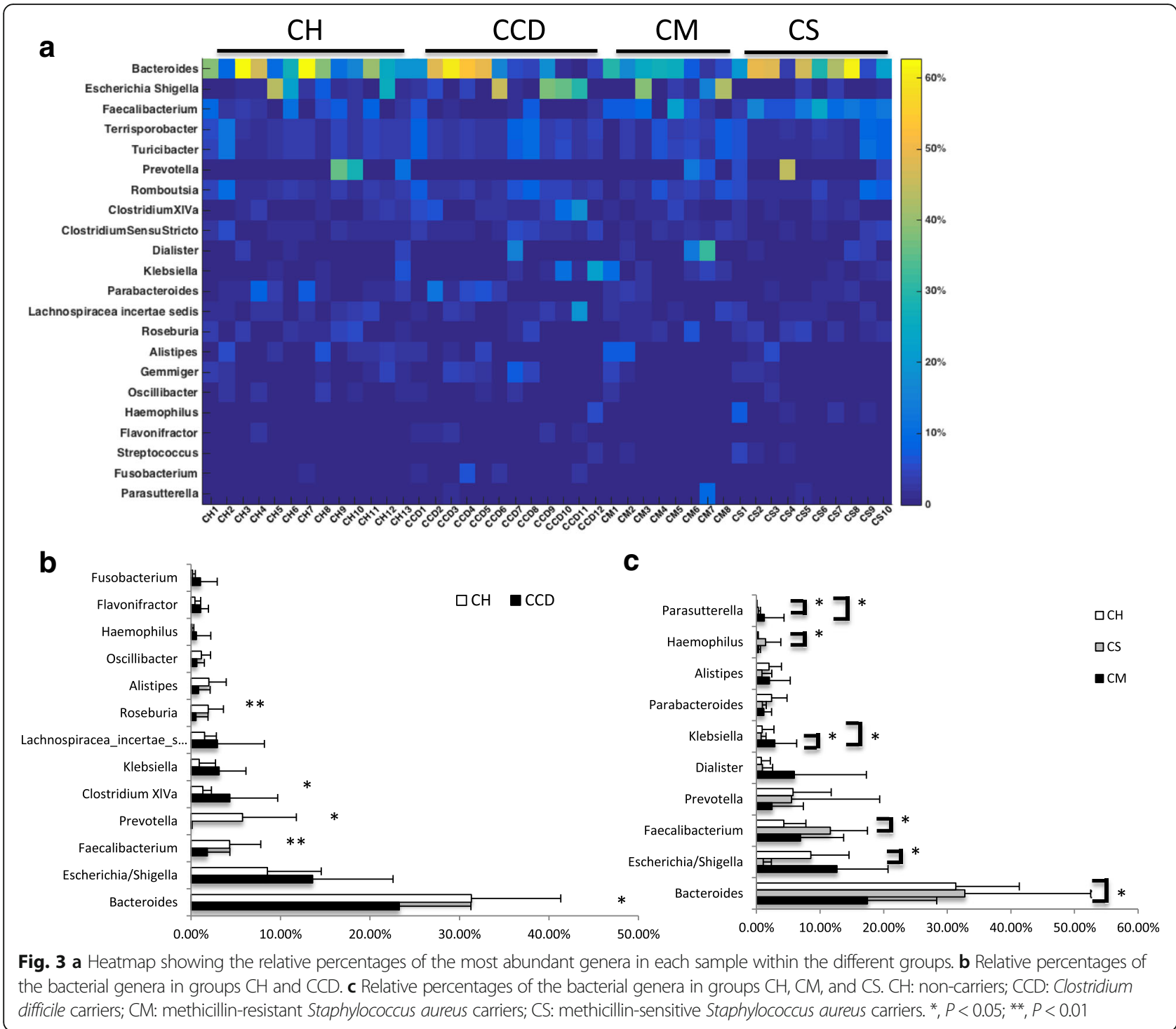

meta-analysis were mostly focused on specific populations at high risk of $S$. aureus carriage, rather than those strictly defined as healthy community-based individuals. Regardless, it is noteworthy that the proportion of MRSA among the total S. aureus population in the current study was $13.33 \%$, which was similar to the 10 . $14 \%$ reported in the meta-analysis. Previous studies have shown that $S$. aureus can colonize the human intestine at any ages from birth, but that the colonization frequency appears to drop later in the life [19]. However, no consensus on this observation has been achieved. Herein, we found that the $S$. aureus carriage rates declined with age, with the prevalence rates in youth twice as high as those in the elderly. This trend was in complete contrast to the colonization pattern observed for $C$. difficile. However, the association between $S$. aureus carriage and age needs to be further investigated.
The human gut is a complex ecosystem inhabited by trillions of commensal bacterial, providing a healthy microenvironment for uptake of essential nutrients, metabolism, and immune protection, as well as pathogen resistance [20]. Alterations in the normal intestinal microbiota often impair colonization resistance, thus allowing for the establishment and proliferation of enteric pathogens such as $C$. difficile and $S$. aureus [8]. Microbial communities in C. difficile-positive and MRSA-infected patients from healthcare facilities always show a marked reduction in bacterial richness and diversity [21]. However, the intestinal microbiota of hospitalized patients can be influenced by various factors, including antibiotics and underlying disease. In our study, in healthy individuals from the community, we did not observe any significant changes in species diversity or richness in the gut microbiota of $C$. difficile or $S$. aureus carriers compared with the controls, and no differences in community structure 
were found by principal coordinate analysis. These findings suggested that colonization by the two pathogens did not significantly alter the microenvironment balance. Host factors such as immune status or comorbidities may affect the integrity of the intestinal microbiota, which could potentially explain the different findings regarding microbial richness in hospitalized and community-based populations.

Although the species richness and diversity in the intestines of the C. difficile and S. aureus carriers were similar to those of non-carriers, there were differences in the community composition between the carriers and non-carriers. Imbalances in the composition of commensal bacterial populations can predispose individuals to infection inflammatory diseases, including inflammatory bowel disease [22]. In accordance with findings regarding $C$. difficile infection and asymptomatic patients by Zhang et al. [21], we observed a lower level of Bacteroidetes and a higher prevalence of Proteobacteria in $C$. difficile and MRSA carriers, implying that species belonging to these two bacterial phyla are sensitive to the presence of $C$. difficile and MRSA, but not MSSA. Interestingly, eradication of Proteobacteria and restoration of Bacteroidetes are considered predictors for successful fecal microbiota transplant [23], emphasizing the importance of balance amongst commensal bacteria. Infection or colonization with $C$. difficile is usually accompanied by depletion or expansion of numerous species related to protein digestion or inflammatory regulation, triggering the overgrowth of enteric pathogens [24]. In our study, the gut populations of $C$. difficile carriers showed a depletion of butyrate-producing bacterial genera Roseburia and Faecalibacterium, as well as Bacteroidetes genera Bacteroides and Prevotella. Butyrate is an energy source for colonocytes, and as such plays a pivotal role in the regulation of colonocyte differentiation, which is important in maintaining intestinal epithelial integrity [25]. Thus, butyrate-producing bacteria may enhance colonic defense barriers by producing antimicrobial peptides, protecting the host from infection. The observed paucity of Roseburia and Faecalibacterium in healthy $C$. difficile carriers in the current study implies that these two genera could be used to represent $C$. difficile-sensitive butyrate-producing bacteria. Like butyrate-producing bacteria, Bacteroides and Prevotella species, which are frequently decreased in patients with $C$. difficile infection or colonization, protect the host against pathogen-mediated colitis inflammation by digesting carbohydrates and producing essential substrates for colonocytes [26]. However, despite only a small subset of genera being affected by $C$. difficile colonization in healthy community-based individuals, our findings did suggest that the gut microbiota of $C$. difficile carriers was more susceptible to further infection.

The gut microbiota signatures of $S$. aureus carriers have not been well determined. Our findings showed that dysbiosis was not present in the gut microbiota of MSSA carriers with regards to either community biodiversity or composition at the phylum level. Although some genera showed differences in, e.g., Haemophilus, Faecalibacterium, and Escherichia/Shigella, which might be related to the presence of MSSA, we could not define the specific role of the changes in terms of microbiota status. Zhao et al. showed that patients with MRSA infection had specific gut microbiota features, exhibiting reduced diversity and decreased prevalence of short-chain fatty acid producers compared with MRSAnegative patients [9]. In our group of healthy communitybased adults, the reduction of Bacteroides species in MRSA carriers was in accordance with the previous report, but no alteration in the prevalence of butyrate-producers was observed. However, the progressively increased proportion of Parasutterella species in MSSA and then MRSA samples was noteworthy. Parasutterella (phylum Proteobacteria) is a relatively recently described genus that is enriched in individuals with Crohn's disease [27]. Klebsiella, another opportunistic enteric bacterial genus, was the only genus showing increased prevalence in MRSA carriers than in MSSA carriers. Antibiotic usage is reported to significantly enhance the proportion of Klebsiella in the gut [28], indicating that cocolonization by MRSA and Klebsiella might be related to exposure to antibiotics in the gut microenvironment. However, because of the small sample size used in the present study, more research needs to be carried out to identify specific genera associated with MRSA or MSSA carriage.

\section{Conclusion}

In conclusion, this study examined the overall prevalence of C. difficile, MSSA, and MRSA in healthy community-based adults in China, providing basic epidemiological data concerning these clinically important pathogens. In this healthy population, the presence of $C$. difficile or $S$. aureus did not significantly alter the community diversity or richness, with only a small set of genera showing changes in prevalence. However, $C$. difficile carriers exhibited a decrease in the prevalence of Bacteroidetes and butyrate-producing genera, indicating a predisposition to further infection. In addition, the specific role of the altered genera in MRSA- and MSSApositive samples should be further investigated. Taken together, our findings would help to better understand the characteristics of gut microbiota in C. difficile and S. aureus carriers in a community setting, providing necessary data for future surveillance and furthering our knowledge of the development of infection.

\section{Additional files}

Additional file 1: Table S1. Detailed information on participants selected for microbiota analysis. (DOCX $42 \mathrm{~kb}$ )

Additional file 2: Figure S1. Rarefaction curves for each sample and OTU numbers of each group. Figure S2. Principal coordinate analysis (PCOA) of bacterial communities using unweighted UniFrac distances of $16 \mathrm{~S}$ rRNA gene sequences. (PPTX $521 \mathrm{~kb}$ ) 


\section{Abbreviations}

MRSA: Methicillin-resistant S. aureus; MSSA: Methicillin-sensitive S. aureus; OTU: Operational taxonomic unit

\section{Acknowledgements}

We thank Tamsin Sheen, PhD, from Liwen Bianji, Edanz Editing China (www.liwenbianji.cn/ac), for editing the English text of a draft of this manuscript.

\section{Funding}

This project was supported by the National Natural Science Foundation of China (grant no. 81572053) and the Research Project of Science and Technology Commission of Shanghai (grant no. 15YF1407300). The funding body had no role in the design of the study and collection, analysis, and interpretation of data and in writing the manuscript.

\section{Availability of data and materials}

All data in this study are available from the corresponding author.

\section{Authors' contributions}

DD, QN and YP designed and executed experiments, interpreted data, and prepared the manuscript. EM designed, interpreted data, and prepared the manuscript. CW, LZ, ZL, and CJ performed experiments and analyzed the data. All authors have read and approved the final manuscript.

\section{Ethics approval and consent to participate}

The Ruijin Hospital Ethics Committee approved the study protocol and obtaining informed consent verbally, because this study only involved patients stool samples and all data collected were anonymized. All participants provided verbal consent prior to participation.

\section{Competing interests}

The authors declare that they have no competing interests.

\section{Publisher's Note}

Springer Nature remains neutral with regard to jurisdictional claims in published maps and institutional affiliations.

\section{Author details}

'Department of Laboratory Medicine, Ruijin Hospital, Shanghai Jiaotong University School of Medicine, No.197 Ruijin ER Road, Shanghai 200025, China. ${ }^{2}$ Department of Laboratory Medicine, Xinhua Hospital, Shanghai Jiaotong University School of Medicine, No. 1665 Kongjiang Road, Shanghai 200092, China. ${ }^{3}$ Department of Laboratory Medicine, Longhua Hospital, Shanghai University of Traditional Medicine, No725 South Wanping Road, Shanghai 200032, China. ${ }^{4}$ Department of Emergency Intensive Care Unit, Ruijin Hospital, Shanghai Jiaotong University School of Medicine, No.197 Ruijin ER Road, Shanghai 200025, China.

Received: 19 January 2018 Accepted: 25 April 2018

\section{Published online: 03 May 2018}

\section{References}

1. Donskey CJ. The role of the intestinal tract as a reservoir and source for transmission of nosocomial pathogens. Clin Infect Dis. 2004;39(2):219.

2. Riggs MM, Sethi AK, Zabarsky TF, Eckstein EC, Jump RL, Donskey CJ. Asymptomatic carriers are a potential source for transmission of epidemic and nonepidemic Clostridium difficile strains among long-term care facility residents. Clin Infect Dis. 2007:45(8):992.

3. Rupnik M, Wilcox MH, Gerding DN. Clostridium difficile infection: new developments in epidemiology and pathogenesis. Nat Rev Microbiol. 2009; 7(7):526

4. Bhalla A, Aron DC, Donskey CJ. Staphylococcus aureus intestinal colonization is associated with increased frequency of S aureus on skin of hospitalized patients. BMC Infect Dis. 2007;7:105.

5. Boyce JM, Havill NL, Otter JA, Adams NM. Widespread environmental contamination associated with patients with diarrhea and methicillinresistant Staphylococcus aureus colonization of the gastrointestinal tract. Infect Control Hosp Epidemiol. 2007;28(10):1142.

6. Vollaard EJ, Clasener HA. Colonization resistance. Antimicrob Agents Chemother. 1994;38(3):409.
7. Hartstra AV, Nieuwdorp M, Herrema H. Interplay between gut microbiota, its metabolites and human metabolism: dissecting cause from consequence. Trends Food Sci Technol. 2016; https://doi.org/10.1016/j.tifs.2016.08.009.

8. Vincent C, Manges AR. Antimicrobial use, human gut microbiota and Clostridium difficile colonization and infection. Antibiotics (Basel). 2015;4(3):230.

9. Zhao J, Nian L, Kwok LY, Sun T, Zhao J. Reduction in fecal microbiota diversity and short-chain fatty acid producers in methicillin-resistant Staphylococcus aureus infected individuals as revealed by PacBio single molecule, real-time sequencing technology. Eur J Clin Microbiol Infect Dis. 2017;36:1463.

10. Wei Y, Gong J, Zhu W, Guo D, Gu L, Li N, et al. Fecal microbiota transplantation restores dysbiosis in patients with methicillin resistant Staphylococcus aureus enterocolitis. BMC Infect Dis. 2015;15:265.

11. CLSI. Performance standards for antimicrobial susceptibility testing; twentyfirst informational supplement, CLSI document m100-S27. Wayne, PA: Clinical and Laboratory Standards Institute; 2017.

12. Yan D, Chen Y, Lv T, Huang Y, Yang J, Li Y, et al. Clostridium difficile colonization and infection in patients with hepatic cirrhosis. J Med Microbiol. 2017;66(10):1483-8.

13. Zheng $Y$, Luo $Y$, LV Y, Huang C, Sheng Q, Zhao P, et al. Clostridium difficile colonization in preoperative colorectal cancer patients. Oncotarget. 2017; 8(7):11877-86

14. Miyajima F, Roberts $P$, Swale A, Price V, Jones M, Horan M, et al. High prevalence of Clostridium difficile colonization among nursing home residents in Hesse. Germany. 2012:7:e30183.

15. Rea MC, O'Sullivan O, Shanahan F, O'Toole PW, Stanton C, Ross RP, et al. Clostridium difficile carriage in elderly subjects and associated changes in the intestinal microbiota. J Clin Microbiol. 2012;50:867.

16. Miyajima F, Roberts $P$, Swale A, Price $V$, Jones $M$, Horan $M$, et al. Characterisation and carriage ratio of Clostridium difficile strains isolated from a community-dwelling elderly population in the United Kingdom. PLoS One. 2011:6:e22804.

17. Verhoeven PO, Gagnaire J, Botelho-Nevers E, Grattard F, Carricajo A, Lucht F, et al. Detection and clinical relevance of Staphylococcus aureus nasal carriage: an update. Expert Rev Anti-Infect Ther. 2014;12:75

18. Gagnaire J, Verhoeven PO, Grattard F, Rigaill J, Lucht F, Pozzetto B, et al. Epidemiology and clinical relevance of Staphylococcus aureus intestinal carriage: a systematic review and meta-analysis. Expert Rev Anti-Infect Ther. 2017:15:767.

19. Acton DS, Plat-Sinnige MJ, van Wamel W, de Groot N, van Belkum A. Intestinal carriage of Staphylococcus aureus: how does its frequency compare with that of nasal carriage and what is its clinical impact? Eur J Clin Microbiol Infect Dis. 2009:28:115.

20. Britton RA, Young VB. Role of the intestinal microbiota in resistance to colonization by Clostridium difficile. Gastroenterology. 2014;146:1547.

21. Zhang L, Dong D, Jiang C, Li Z, Wang X, Peng Y. Insight into alteration of gut microbiota in Clostridium difficile infection and asymptomatic $C$ difficile colonization. Anaerobe. 2015:34:1.

22. Buffie CG, Pamer EG. Microbiota-mediated colonization resistance against intestinal pathogens. Nat Rev Immunol. 2013; https://doi.org/10.1038/nri3535.

23. Seekatz AM, Aas J, Gessert CE, Rubin TA, Saman DM, Bakken JS, et al. Recovery of the gut microbiome following fecal microbiota transplantation. MBio. 2014;5:e00893.

24. Lawley TD, Clare S, Walker AW, Goulding D, Stabler RA, Croucher N, et al. Antibiotic treatment of Clostridium difficile carrier mice triggers a supershedder state, spore-mediated transmission, and severe disease in immunocompromised hosts. Infect Immun. 2009:77.3661.

25. Guilloteau P, Martin L, Eeckhaut V, Ducatelle R, Zabielski R, Van Immerseel F. From the gut to the peripheral tissues: the multiple effects of butyrate. Nutr Res Rev. 2010;23:366

26. Bibbo S, Lopetuso LR, laniro G, Di Rienzo T, Gasbarrini A, Cammarota G, et al. Role of microbiota and innate immunity in recurrent infection. J Immunol Res. 2014:2014:462740.

27. Chiodini RJ, Dowd SE, Chamberlin WM, Galandiuk S, Davis B, Glassing A. Microbial population differentials between mucosal and submucosal intestinal tissues in advanced Crohn's disease of the ileum. PLoS One. 2015:10:e0134382.

28. Orrhage K, Brismar B, Nord CE. Effect of supplements with bifidobacteriurn longum and lactobacillus acidophilus on the intestinal microbiota during administration of clindamycin. Microb Ecol Health Dis. 1994;7:17. 